\title{
Reverse remodeling and the mechanism of mitral regurgitation improvement in patients with dilated cardiomyopathy
}

Rafael Kuperstein ${ }^{1,2}$, Ido Blechman ${ }^{1,2}$, Sagit Ben Zekry ${ }^{1,2}$, Robert Klempfner ${ }^{1,2}$, Dov Freimark ${ }^{1,2}$, Michael Arad ${ }^{1,2}$

${ }^{1}$ Leviev Heart Institute, Sheba Medical Center, Sheba, Israel

${ }^{2}$ Sackler School of Medicine, Tel-Aviv University, Tel-Aviv, Israel

\begin{abstract}
Background: Functional mitral regurgitation (MR) is a common finding in dilated cardiomyopathy. Left ventricular $(L V)$ reverse remodeling with $L V$ size reduction and improvement in $L V$ function is a well recognized phenomenon. We aimed to evaluate the impact of LV remodeling on the mechanism leading to functional MR.

Methods: Among 188 patients with non-ischemic dilated cardiomyopathy, 10 patients significantly improved their $L V$ function, reduced $L V$ size and $M R$ severity during follow-up (RRMR). A comparison was made between their baseline and follow-up echocardiographic examinations and to a matched-control group of patients who did not improve (no RRMR). $L V$ and left atrium (LA) dimensions and volumes, $L V$ mass (LVM), LV ejection fraction (LVEF) (Simpsons), sphericity index (SI), mitral valve tenting area (TA) coaptation distance (CD), effective regurgitant orifice (ERO), and regurgitant volume were calculated. Multivariable analysis was performed in order to evaluate which echocardiographic parameters related to $M R$ improvement in reverse remodeling.

Results: $L V$ and $L A$ dimensions and volumes, $L V M, S I, T A, C D, E R O$ and right ventricle, in the RRMR group significantly decreased at follow-up ( $p<0.04$ for all). When compared to no $R R M R$, despite a similar ERO (0.2 \pm 0.05 vs. $0.2 \pm 0.08, p=0.13)$ and a larger regurgitant volume (38 \pm 9 vs. $29 \pm 8 \mathrm{~mL}, p=0.05)$ and despite similar clinical characteristics and medical treatment we found significantly higher $L V E F$, smaller $L V$ dimensions and volumes, smaller LVM and SI in the RRMR group ( $p<0.05$ for all). On multivariable analysis the SI was the sole predictor of RRMR $(p=0.04, O R=0.76, C I 0.58-0.99)$.

Conclusions: Reverse remodeling characterized by improvement in LV function, reduction in $L V$ size and an associated reduction in MR severity is related to LV SI at baseline. (Cardiol J 2015; 22, 4: 391-396)
\end{abstract}

Key words: dilated cardiomyopathy, mitral regurgitation, reverse remodeling

\section{Introduction}

Dilated cardiomyopathy (DCM) is a syndrome characterized by cardiac enlargement and impaired systolic function of one or both ventricles [1, 2]. Functional mitral regurgitation (MR) is a common finding in patients with DCM resulting from dilation of the annulo-ventricular apparatus, the

Address for correspondence: Dr Rafael Kuperstein, MD, Leviev Heart Institute, Sheba Medical Center, Tel Hashomer 52621, Israel, tel: +972-502039087, fax: +972-03-5302433, e-mail: rafikupe@gmail.com 
increased sphericity of ventricular geometry and apico-lateral displacement of the papillary muscles $[3,4]$. MR exacerbates the volume overload of the already dilated left ventricle (LV), leading to progressive ventricular and annular dilation, heart failure deterioration and predicts a poor survival [5-7]. Reverse remodeling, a well recognized phenomenon characterized by a decrease in LV size in association with normalization of LV shape and improvement in LV function, occurs in about a third of DCM patients under contemporary treatment [8]. This process is sometimes associated with a variable reduction in the severity of MR. In the current study we evaluated the mechanisms associated with improvement of MR in patients with DCM who reverse remodeled and significantly reduced their MR.

\section{Methods}

\section{Echocardiographic examination}

Serial echo-Doppler studies were performed every 6-24 months or as clinically indicated. LV and left atrial (LA) dimensions were measured according to the American Society of Echocardiography guidelines for chamber quantification [9] Right ventricular (RV) fractional area change was calculated from the apical 4-chamber view. LV end-systolic and end-diastolic volumes (LVESV, LVEDV) and LV ejection fraction (LVEF) function were measured according to the Simpsons' method of discs averaging 3 consecutive beats. LA volume was measured using the Simpson's method from the 4 chamber apical view, indexed to body surface area (BSA), LA volume index (LAVi). Care was taken to exclude the pulmonary veins from the LA tracing. LV mass (LVM) was measured according to the Devereux formula and indexed to BSA (LVMi). In order to assess changes in LV shape, the sphericity index (SI) was calculated as the short to long axis ratio in the 4 chamber view. Tenting area was defined as the area enclosed between the annular plane and the mitral leaflets from the parasternal long axis view at mid-systole. Coaptation distance was defined as the distance between the annular plane and the coaptation point at mid systole in the 4 chamber apical view. The effective regurgitant orifice and the regurgitant volume were calculated according to the PISA method and the MR was graded on 4 point scale, minimal or none/ /minimal -0 , mild -1 , moderate -2 , severe - 3. Improvement in $M R \geq 1$ grade was defined as significant. Reverse remodeling of the LV was defined as an increase in LVEF by at least $10 \%$ units concomitant with a decrease in the LV end-diastolic dimension (LVEDD) index by at least $10 \%$ relative to the baseline measurement.

\section{Patient population}

The Sheba Heart Failure clinic is a tertiary center taking care of heart failure patients. As we previously reported [10], our clinical database includes 233 patients with DCM examined between 2004 and 2008 with subsequent follow-up. One hundred and eighty-eight patients with mean age $58 \pm 16$ years (range 11-93 years), 115 (61\%) male, LVEF $28 \pm 9 \%$ (range 10-48\%), LVEDD $60 \pm 8 \mathrm{~mm}$ (range 42-88 $\mathrm{mm}$ ), LV end-systolic dimension (LVESD) $47 \pm 9 \mathrm{~mm}$ (range 28-82 $\mathrm{mm}$ ) had a complete follow-up (mean follow-up time $82 \pm 35$ months). Seventy-three patients were hypertensive and 42 diabetic. The etiology was familial in 37 , post-chemotherapy in 18 , pregnancy-related in 19 and probably tachycardia-induced in 25 patients. Nineteen patients had conduction system disease and 11 patients declared about previous substance abuse. The etiology was idiopathic in the remaining patients. Patients with associated significant coronary artery disease (ruled out by coronary angiography or radionuclide scan) and valvular heart disease were not included in the database. Ninety-three patients did not improve their LVEF by $\geq 10 \%$ and did not decrease their LVEDD index by $\geq 10 \%$ during follow-up, 37 patients improved their LVEF without decreasing their LVEDD, 8 patients reduced their LVEDD without improving their LVEF while 50 patients improved their LVEF and reduced LVEDD index during the follow-up. The prevalence of $\geq$ grade $2 \mathrm{MR}$ was similar among the groups $(p=0.1)$, while improvement in the severity of MR at follow-up was significantly better in the patients who improved LVEF and reduced their LVEDD index ( $p=0.008$, Fig. 1$)$.

Twelve of the 50 patients who improved LVEF and reduced their LVEDD index had at least moderate MR at baseline and significantly reduced their MR severity by $\geq 1$ grade at longterm follow-up. Ten of them had good quality follow-up echocardiograms available for evaluation (RRMR group). Their baseline echocardiographic examinations were compared to their follow-up echo examinations (after improvement) looking for the morphologic changes related to the reduction in MR severity. Thereafter, their first echo examinations were compared to the first echo examinations of a matched control group of patients with similar LV dysfunction and MR severity at baseline who did not reverse remodel 


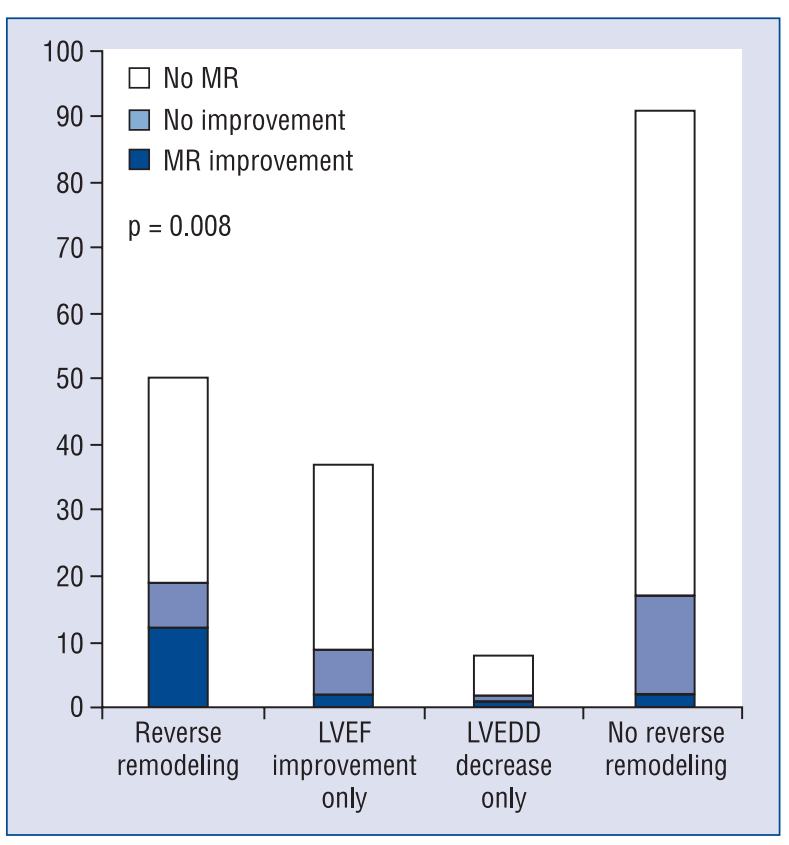

Figure 1. Prevalence of improvement in the severity of mitral regurgitation (MR) according to the pattern of left ventricular conformational changes at follow-up. There was no difference in the prevalence of significant MR among the groups $(p=0.1)$ but improvement in MR severity was more common in patients with reverse remodeling ( $p=0.008)$; LVEF — left ventricular ejection fraction; LVEDD - left ventricular end-diastolic dimension.

their LV and did not improve their MR during the follow-up (no RRMR group).

The study protocol was approved by our institutional ethic committee for human research.

\section{Statistical methods}

Baseline and follow-up echocardiographic values were compared using the paired sample t-test or the Wilcox signed-rank test, as appropriate. The echocardiographic characteristics of the patients who improved LV function and reduced MR severity (RRMR) were compared to matched patients that did not improve LV function or reduce MR severity (no RRMR). Comparison of categorical variables was performed with $\chi^{2}$ analysis and comparison of continuous variables was performed with the student's t-test for variables with normal distribution and by Kruskal-Wallis for those that violated the normality assumption.

In order to evaluate the independent association of baseline echocardiographic parameters with the probability of RRMR we selected co-variates that were associated with RRMR in a univariable level, at a significance level of $\leq 0.01$. The univariable association of each echocardiographic parameter, LA dimesion $(\mathrm{mm})$, LA area $\left(\mathrm{cm}^{2}\right), \mathrm{LAVi}\left(\mathrm{mL} / \mathrm{m}^{2}\right)$, RV-fractional area change (\%), LVEDD (mm), LVESD ( $\mathrm{mm})$, LVEDV $\left(\mathrm{cm}^{3}\right)$, LVESV $\left(\mathrm{cm}^{3}\right), \operatorname{LVEF}$ (\%), LVM (g), LVMi $\left(\mathrm{g} / \mathrm{m}^{2}\right)$, effective regurgitant orifice $\left(\mathrm{mm}^{2}\right)$, regurgitant volume $(\mathrm{mL})$, SI, coaptation distance $(\mathrm{cm})$, tenting area $\left(\mathrm{cm}^{2}\right)$, with the RRMR outcome, was separately assessed using a binary logistic regression model. Co-variates were introduced using the enter method, and the adjusted odds ratio are presented with their $95 \%$ confidence interval (CI).

All $p$ values were 2 -sided, and a $p$ value $\leq 0.05$ was considered significant. The statistical software used was SPSS version 20 (IBM Inc.).

\section{Results}

The baseline echocardiographic findings of the RRMR patients were compared with their findings after the follow-up. Effective regurgitant orifice decreased from $0.2 \pm 0.05$ to $0.01 \pm 0.03$, $\mathrm{p}<0.0001$. The regurgitant volume was $38 \pm 9 \mathrm{~mL}$ at admission and was immeasurable (due to insignificant MR) in most patients at follow-up. The RV area changes significantly increased from $45 \pm 5 \%$ at baseline to $52 \pm 9 \%$ at follow-up ( $\mathrm{p}=$ $=0.03)$. LA and LV dimensions, LVM, LVMi (LVM/ /body surface area), LVESV and LVEDV, LAVi, SI, mitral valve tenting area and coaptation distance were all significantly smaller at follow-up after reverse remodeling (Table 1 ). In Table 2 , the baseline clinical characteristics of the 10 patients with reverse remodeling (RRMR) are compared with 10 matched controls who did not reverse remodel at follow-up (no RRMR). No differences were found between the groups. In Table 3 , the baseline echocardiographic characteristics of the matched groups are compared. The effective regurgitant orifice was similar in both groups $(0.2 \pm$ \pm 0.05 and $0.2 \pm 0.08, \mathrm{p}=0.13$ ) respectively, while the regurgitant volume was significantly higher in the RRMR group than in the no RRMR group (38 $\pm 9 \mathrm{~mL}$ vs. $29 \pm 8 \mathrm{~mL}, \mathrm{p}=0.05)$. When compared to the no RRMR group baseline LA dimensions, LAVi, LV dimensions, LVESV and LVEDV, LVM, LVMi and SI were all significantly smaller in the group who reverse remodeled at follow-up. On univariable analysis baseline LAVi, LVESV and SI where associated with RRMR ( $p<0.01$ for each). On multivariable analysis SI remained as the sole predictor of RRMR ( $\mathrm{p}=0.04$, CI 0.58-0.99). 
Table 1. Baseline and follow-up echocardiographic dimensions in patients who reverse remodeled and reduced mitral regurgitation $(\mathrm{n}=10)$.

\begin{tabular}{lccc}
\hline & Baseline & Follow-up & P \\
\hline Left atrial dimension $[\mathrm{mm}]$ & $42 \pm 4$ & $37 \pm 6$ & 0.02 \\
Left atrial area $\left[\mathrm{cm}^{2}\right]$ & $24 \pm 4$ & $21 \pm 6$ & 0.3 \\
LAVi $\left[\mathrm{mL} / \mathrm{m}^{2}\right]$ & $35 \pm 6$ & $27 \pm 9$ & 0.04 \\
RV-FAC $[\%]$ & $45 \pm 5$ & $52 \pm 9$ & 0.03 \\
LVEDD [mm] & $57 \pm 2$ & $48 \pm 5$ & $>0.001$ \\
LVESD [mm] & $45 \pm 6$ & $31 \pm 4$ & $>0.001$ \\
LVEDV $\left[\mathrm{cm}^{3}\right]$ & $135 \pm 37$ & $94 \pm 24$ & 0.006 \\
LVESV $\left[\mathrm{cm}^{3}\right]$ & $98 \pm 34$ & $48 \pm 13$ & $>0.001$ \\
LVEF $[\%]$ & $28 \pm 10$ & $48 \pm 10$ & $>0.001$ \\
LVM $[\mathrm{g}]$ & $231 \pm 38$ & $187 \pm 49$ & 0.02 \\
LVM index $\left[\mathrm{g} / \mathrm{m}^{2}\right]$ & $124 \pm 15$ & $100 \pm 22$ & 0.02 \\
ERO $\left[\mathrm{mm}{ }^{2}\right]$ & $0.2 \pm 0.05$ & $0.01 \pm 0.03$ & $<0.0001$ \\
Regurgitant volume $[\mathrm{mL}]$ & $38 \pm 9$ & Not measurable & $<0.0001$ \\
Sphericity index & $0.56 \pm 0.07$ & $0.5 \pm 0.07$ & 0.04 \\
Coaptation distance $[\mathrm{cm}]$ & $0.79 \pm 0.23$ & $0.48 \pm 0.14$ & $>0.001$ \\
Tenting area $\left[\mathrm{cm}^{2}\right]$ & $2.53 \pm 0.52$ & $1.48 \pm 0.4$ & 0.003 \\
\hline
\end{tabular}

LAVi — left atrial volume/body surface area; RV-FAC — right ventricular fractional area change, LVEDD — left ventricular end-diastolic diameter; LVESD - left ventricular end-systolic diameter; LVEDV — left ventricular end-diastolic volume, LVESV — left ventricular end-systolic volume; LVEF — left ventricular ejection fraction; LVM — left ventricular mass; ERO — effective regurgitant orifice

Table 2. Baseline clinical characteristics of the matched groups.

\begin{tabular}{lccc} 
& RRMR & No RRMR & P \\
\hline No. of patients & 10 & 10 & 0.9 \\
Age [years] & $61 \pm 17$ & $60 \pm 20$ & 0.6 \\
Male sex & $5(50 \%)$ & $6(60 \%)$ & 0.7 \\
BMI $\left[\mathrm{kg} / \mathrm{m}^{2}\right]$ & $26 \pm 3$ & $27 \pm 8$ & 0.7 \\
BSA $\left[\mathrm{m}^{2}\right]$ & $1.86 \pm 0.19$ & $1.82 \pm 0.25$ & 0.3 \\
Familial CMP $[\%]$ & $0(0 \%)$ & $1(10 \%)$ & 0.6 \\
Hypertension & $5(50 \%)$ & $4(40 \%)$ & 0.3 \\
Diabetes & $3(30 \%)$ & $1(10 \%)$ & 0.2 \\
Renal failure & $5(50 \%)$ & $2(20 \%)$ & 0.08 \\
Mineralocorticoid antagonist & $3(30 \%)$ & $7(70 \%)$ & 0.556 \\
ACEl/ARB & $8(80 \%)$ & $9(90 \%)$ & 0.151 \\
Beta-blocker & $8(80 \%)$ & $10(100 \%)$ & \\
\hline
\end{tabular}

RRMR - patients who improved left ventricular function, reduced left ventricular end-diastolic dimension (LVEDD) and reduced mitral regurgitation severity at follow-up; No RRMR - patients who did not improve left ventricular function, did not reduce LVEDD and did not reduce mitral regurgitation severity at follow-up; BMI — body mass index; BSA — body surface area; CMP — cardiomyopathy; ACEI — angiotensin converting enzyme inhibitors; ARB — angiotensin receptor blocker

\section{Discussion}

The term DCM refers to a large group of heterogeneous myocardial disorders that are characterized by ventricular dilation and depressed myocardial contraction in the absence of abnormal loading conditions [1,2]. Some DCM patients have a relatively benign clinical course and spontaneously improve symptoms and partially or completely recover their LV function [8]. Functional MR is a frequent finding in patients with DCM and its severity is associated with prognosis [5-7]. The 
Table 3. Baseline echocardiographic characteristics of the matched groups.

\begin{tabular}{|c|c|c|c|}
\hline & $\operatorname{RRMR}(n=10)$ & No RRMR $(n=10)$ & $\mathbf{P}$ \\
\hline Left atrial dimension [mm] & $42 \pm 4$ & $49 \pm 5$ & 0.005 \\
\hline Left atrial area $\left[\mathrm{cm}^{2}\right]$ & $24 \pm 4$ & $30 \pm 7$ & 0.025 \\
\hline LAVi $\left[\mathrm{mL} / \mathrm{m}^{2}\right]$ & $35 \pm 6$ & $49 \pm 16$ & 0.03 \\
\hline RV-FAC [\%] & $45 \pm 5$ & $42 \pm 10$ & 0.4 \\
\hline LVEDD [mm] & $57 \pm 2$ & $67 \pm 9$ & 0.002 \\
\hline LVESD $[\mathrm{mm}]$ & $45 \pm 6$ & $58 \pm 9$ & 0.002 \\
\hline LVEDV $\left[\mathrm{cm}^{3}\right]$ & $135 \pm 37$ & $188 \pm 61$ & 0.03 \\
\hline LVESV $\left[\mathrm{cm}^{3}\right]$ & $98 \pm 34$ & $150 \pm 55$ & 0.02 \\
\hline LVEF [\%] & $28 \pm 10$ & $21 \pm 4$ & 0.05 \\
\hline LVM [g] & $231 \pm 38$ & $318 \pm 121$ & 0.04 \\
\hline LVM index $\left[\mathrm{g} / \mathrm{m}^{2}\right]$ & $124 \pm 15$ & $177 \pm 78$ & 0.05 \\
\hline $\mathrm{ERO}\left[\mathrm{mm}^{2}\right]$ & $0.2 \pm 0.05$ & $0.2 \pm 0.08$ & 0.13 \\
\hline Regurgitant volume $[\mathrm{mL}]$ & $38 \pm 9$ & $29 \pm 8$ & 0.05 \\
\hline Sphericity index & $0.56 \pm 0.07$ & $0.66 \pm 0.05$ & 0.003 \\
\hline Coaptation distance $[\mathrm{cm}]$ & $0.79 \pm 0.23$ & $0.95 \pm 0.38$ & 0.2 \\
\hline Tenting area $\left[\mathrm{cm}^{2}\right]$ & $2.53 \pm 0.52$ & $2.14 \pm 0.48$ & 0.1 \\
\hline
\end{tabular}

Abbreviations as in Tables 1 and 2.

pathophysiology of functional MR has been widely investigated. Functional MR develops because of a combination of mitral leaflet tethering secondary to LV dilatation/deformation with papillary displacement/discoordination, annular dilatation/ /dysfunction, insufficient LV-generated closing forces attributable to reduction of LV contractility, and global LV/papillary muscle dyssynchrony [11, 12]. Tethering of the mitral leaflets is the principal lesion of functional MR and results in restriction of systolic leaflet motion functional MR does not typically occur in global LV dysfunction without tethering. However, once tethering occurs, leaflet closure is further impaired by LV dysfunction because of decreased force opposing tethering [13-16]. The key event in the pathogenesis of functional MR is the distortion of normal LV geometry (LV remodeling) with subsequent apical and lateral displacement of papillary muscles, which, in turn, draws the chordae tendineae away from the line of coaptation $[14,15]$. In this study, a significant reduction in the severity of MR occurred in $24 \%$ of the patients who underwent reverse remodeling, i.e. improved their LV function and concomitantly decreased their LV diastolic dimensions. Disappearance of MR was much less prevalent in patients who improved only one of these variables (Fig. 1). Our findings suggest that the improvement in MR in patients with DCM who reverse remodeled is related to the reduction of $\mathrm{LV}$ dimensions and changes in LV shape but is determined by the LV shape at baseline. This reduction in LVESV and LVEEV, as well as the reduction in the SI translate into a reduction in the coaptation distance and in tenting area with a consequent reduction in the severity of MR depending on the SI at baseline so that the higher the SI at baseline the worse the chances are of reverse remodeling. In this study, per each 0.01 increment in the baseline SI the odds ratio for reverse remodeling was reduced by $24 \%$. The practical implication is that mitral valve apparatus acts as a "marionette" of LV conformational changes [17], but the chance of reverse remodeling is related to the $\mathrm{LV}$ conformational changes at baseline (Table 3 ). Importantly, the SI was only mildly affected by the process of RRMR (Table 1) and thus emerged as a fundamental property of the LV affecting prognosis. Actually, an ellipsoid shape is vital for optimal LV function. In pathological disease states such as DCMs there is a loss of the obliquity of the cardiac myofibers leading to a more globular LV shape which initiate the cascade of heart failure [18]. The comparison of a group of patients with reverse remodeling and significant MR reduction to a group of patients who did not reverse remodel showed similar clinical characteristics at baseline. Patients who did not reduce their LV dimensions or recover their LV shape did not improve their MR. 
Importantly, similar changes in LV geometry and LV contraction have been described and found to be associated with improvement in functional MR severity after cardiac resynchronization therapy [19-22]. It remains to be determined how the SI and the capacity to revert MR are related to epidemiological features such as disease duration and etiology.

\section{Limitations of the study}

The current study population was enrolled in a tertiary referral center for cardiomyopathies and heart failure, thus imposing a selection bias with respect to the characteristics of DCM in the general population. It is a small observational study and prospective echo-Doppler follow-up was not available in some patients. The measurements were performed by a single operator (R.K.) who was not blinded to the results of the baseline echocardiographic studies. The baseline effective regurgitant orifice was similar between the RRMR and the no RRMR group but the regurgitant volume was not. This can be explained by the fact that values defining MR severity in individual patients depend on multiple factors including LVEDV, LVEF and the pressure gradient between LA and LV [23]. Finally, the ideal control group for this study would comprise patients who reverse remodeled and did not reduce the severity of their MR. However, this comparison was not possible due to the small number of participants.

\section{Conclusions}

Reverse remodeling is a common finding in DCM under contemporary therapy involving $\sim 1 / 4$ of patients with established disease. Reduction in the severity of functional MR is quite prevalent in patients with DCM who present with significant MR and reverse remodel during follow-up. In patients with less distorted LV shape at baseline a further reduction in $\mathrm{LV}$ dimension in association with changes in LV shape and function translate into less valvular tethering, better leaflet coaptation and improvement in functional MR.

\section{Conflict of interest: None declared}

\section{References}

1. Elliott P, Andersson B, Arbustini E et al. Classification of the cardiomyopathies: a position statement from the European society of cardiology working group on myocardial and pericardial diseases. Eur Heart J, 2008; 29: 270-276.
2. Maron BJ, Towbin JA, Thiene G et al. Contemporary definitions and classification of the cardiomyopathies: an American Heart Association Scientific Statement from the Council on Clinical Cardiology, Heart Failure and Transplantation Committee; Quality of Care and Outcomes Research and Functional Genomics and Translational Biology Interdisciplinary Working Groups; and Council on Epidemiology and Prevention. Circulation, 2006; 113: 1807-1816.

3. Boltwood CM, Tei C, Wong M, Shah PM. Quantitative echocardiography of the mitral complex in dilated cardiomyopathy: The mechanism of functional mitral regurgitation. Circulation, 1983; 68: 498-508.

4. Kono T, Sabbah HN, Rosman H, Alam M, Jafri S, Goldstein S. Left ventricular shape is the primary determinant of functional mitral regurgitation in heart failure. J Am Coll Cardiol, 1992; 7: 1594-1598.

5. Blondheim DS, Jacobs LE, Kotler MN, Costacurta GA, Parry WR Dilated cardiomyopathy with mitral regurgitation: decreased survival despite a low frequency of left ventricular thrombus. Am Heart J, 1991; 3 (part 1): 763-771.

6. Rossi A, Dini FL, Faggiano P et al. Independent prognostic value of functional mitral regurgitation in patients with heart failure. A quantitative analysis of 1256 patients with ischaemic and non-ischaemic dilated cardiomyopathy. Heart, 2011; 97: 1675-1680.

7. Trichon BH, Felker GM, Shaw LK, Cabell CH, O'Connor CM. Relation of frequency and severity of mitral regurgitation to survival among patients with left ventricular systolic dysfunction and heart failure. Am J Cardiol, 2003; 91: 538-543.

8. Merlo M, Pyxaras SA, Pinamonti B, Barbati G, Di Lenarda A, Sinagra G. Prevalence and prognostic significance of left ventricular reverse remodeling in dilated cardiomyopathy receiving tailored medical treatment. J Am Coll Cardiol, 2011; 57: 1468-1476.

9. Lang RM, Bierig M, Devereux RB et al. Recommendations for chamber quantification: A report from the American Society of Echocardiography's guidelines and standards committee and the chamber quantification writing group, developed in conjunction with the European Association of Echocardiography, a brancg of the European Society of Cardiology. J Am Soc Echocard, 2005; 18: 114-163.

10. Arad M, Nussbaum T, Blechman I et al. Prevalence and clinical predictors of reverse remodeling in patients with dilated cardiomyopathy. Isr Med Assoc J, 2014; 7: 405-411.

11. Levine RA, Schwammenthal E. Ischemic mitral regurgitation on the threshold of a solution: from paradoxes to unifying concepts. Circulation, 2005; 112: 745-758.

12. Marwick TH, Lancellotti P, Pierard L. Ischemic mitral regurgitation: Mechanisms and diagnosis. Heart, 2009; 95: 1711.

13. Silbiger JJ. Novel pathogenic mechanisms and structural adaptations in ischemic mitral regurgitation. J Am Soc Echocard, 2013; 26: 1107-1117.

14. Ciarka A, Van de Veire N. Secondary mitral regurgitation: Pathophysiology, diagnosis and treatment. Heart, 2011; 97: 1012-1023.

15. He S, Fonaine AA, Shwammenthal E, Yoganatan AP, Levine RA. Integrated mechanism for functional mitral regurgitation: Leaflet restriction versus coapting force: in vitro studies. Circulation, 1997; 96: $1826-1834$.

16. Otsuji Y, Handshumacher E, Liel-Cohen N et al. Mechanism of ischemic mitral regurgitation with segmental left ventricular dysfunction: Three-dimensional echocardiographic studies in models of acute and chronic progressive regurgitation. K Am Coll Cardiol, 2001; 37: 641-648.

17. Levine RA, Messas E, Nathan NS, Rudski LG. New understanding of ischemic mitral regurgitation: the marionette and its masters. Eur J Echocard, 2004; 5: 313-317.

18. Adhyapak SM, Parachuri VR. Architecture of left ventricle: insights for optimal surgical ventricular restoration. Heart Fail Rev, 2010; 15: 73-83.

19. Ypenburg C, Lancellotti P, Tops LF et al. Mechanism of improvement in mitral regurgitation after cardiac resynchronization therapy. Eur Heart J, 2008; 29: 757-765.

20. Van Bommel RJ, Marsan NA, Delgado V et al. Cardiac resynchronization therapy as a therapeutic option in patients with moderate-severe functional mitral regurgitation and a high operative risk. Circulation, 2011; 124: 912-919.

21. Solis J, McCarty D, Levine RA et al. Mechanism of decrease in mitral regurgitation after cardiac resynchronization therapy: optimization of force-balance relationship. Circ Cardiovasc Imag, 2009; 2: 444-450.

22. Sitges M, Vidal B, Delgado V et al. Long term effects of cardiac resynchronization therapy on functional mitral valve regurgitation. Am J Cardiol, 2009; 104: 383-388.

23. Grayburn P, Carabello B, Hung J et al. Defining severe secondary mitral regurgitation: emphasizing an integrated approach. J Am Coll Cardiol, 2014; 64: 2792-2801. 